PROCEEDINGS OF THE

AMERICAN MATHEMATICAL SOCIETY

Volume 136, Number 12, December 2008, Pages 4223-4233

S 0002-9939(08)09500-2

Article electronically published on July 25, 2008

\title{
DAHLBERG'S BILINEAR ESTIMATE FOR SOLUTIONS OF DIVERGENCE FORM COMPLEX ELLIPTIC EQUATIONS
}

\author{
STEVE HOFMANN \\ (Communicated by Michael T. Lacey)
}

\begin{abstract}
We consider divergence form elliptic operators $L=-\operatorname{div} A(x) \nabla$, defined in $\mathbb{R}^{n+1}=\left\{(x, t) \in \mathbb{R}^{n} \times \mathbb{R}\right\}, n \geq 2$, where the $L^{\infty}$ coefficient matrix $A$ is $(n+1) \times(n+1)$, uniformly elliptic, complex and $t$-independent. Using recently obtained results concerning the boundedness and invertibility of layer potentials associated to such operators, we show that if $L u=0$ in $\mathbb{R}_{+}^{n+1}$, then for any vector-valued $\mathbf{v} \in W_{l o c}^{1,2}$, we have the bilinear estimate

$$
\left|\iint_{\mathbb{R}_{+}^{n+1}} \nabla u \cdot \overline{\mathbf{v}} d x d t\right| \leq C \sup _{t>0}\|u(\cdot, t)\|_{L^{2}\left(\mathbb{R}^{n}\right)}\left(\|t \nabla \mathbf{v}\| \mid+\left\|N_{*} \mathbf{v}\right\|_{L^{2}\left(\mathbb{R}^{n}\right)}\right),
$$

where $\left\||F \|| \equiv\left(\iint_{\mathbb{R}_{+}^{n+1}}|F(x, t)|^{2} t^{-1} d x d t\right)^{1 / 2}\right.$, and where $N_{*}$ is the usual nontangential maximal operator. The result is new even in the case of real symmetric coefficients and generalizes an analogous result of Dahlberg for harmonic functions on Lipschitz graph domains. We also identify the domain of the generator of the Poisson semigroup for the equation $L u=0$ in $\mathbb{R}_{+}^{n+1}$.
\end{abstract}

\section{INTRODUCTION}

In [6, B. Dahlberg considered the bilinear singular integral form

$$
\int_{\Omega} \nabla u \cdot \overline{\mathbf{v}}
$$

where $u$ is harmonic in the domain $\Omega \equiv\left\{(x, t) \in \mathbb{R}^{n+1}: t>\varphi(x)\right\}$, with $\varphi$ Lipschitz, and where $\mathbf{v} \in W_{\text {loc }}^{1,2}$ is vector-valued. He showed that the bilinear form (1.1) is bounded by the $L^{2}$-norm of the square function plus the non-tangential maximal function of $u$, times the same expression for $\mathbf{v}$. In the present note, we generalize Dahlberg's Theorem to variable coefficient divergence form elliptic operators. To be precise, let

$$
L=-\operatorname{div} A \nabla \equiv-\sum_{i, j=1}^{n+1} \frac{\partial}{\partial x_{i}}\left(A_{i, j} \frac{\partial}{\partial x_{j}}\right)
$$

be defined in $\mathbb{R}^{n+1}=\left\{(x, t) \in \mathbb{R}^{n} \times \mathbb{R}\right\}, n \geq 2$ (we use the convention that $\left.x_{n+1}=t\right)$, where $A=A(x)$ is an $(n+1) \times(n+1)$ matrix of complex-valued

Received by the editors April 27, 2007.

2000 Mathematics Subject Classification. Primary 42B20, 42B25, 35J25.

The author was supported by the National Science Foundation.

(C)2008 American Mathematical Society Reverts to public domain 28 years from publication 
$L^{\infty}$ coefficients, defined on $\mathbb{R}^{n}$ (i.e., independent of the $t$ variable), and satisfying the uniform ellipticity (accretivity) condition

$$
\lambda|\xi|^{2} \leq \Re e\langle A(x) \xi, \xi\rangle,\|A\|_{L^{\infty}\left(\mathbb{R}^{n}\right)} \leq \Lambda,
$$

for some $\lambda>0, \Lambda<\infty$, and for all $\xi \in \mathbb{C}^{n+1}, x \in \mathbb{R}^{n}$. Here, $\langle\cdot, \cdot\rangle$ denotes the usual Hermitian inner product in $\mathbb{C}^{n+1}$, so that

$$
\langle A(x) \xi, \xi\rangle \equiv \sum_{i, j=1}^{n+1} A_{i j}(x) \xi_{j} \bar{\xi}_{i} .
$$

In order to state our theorem, we first recall that the non-tangential maximal operator $N_{*}$ (and a variant $\widetilde{N}_{*}$ ) are defined as follows. Given $x_{0} \in \mathbb{R}^{n}$, let

$$
\gamma\left(x_{0}\right)=\left\{(x, t) \in \mathbb{R}_{+}^{n+1}:\left|x_{0}-x\right|<t\right\}
$$

denote the cone with vertex at $x_{0}$. Then for $U$ defined in $\mathbb{R}_{+}^{n+1}$,

$$
\begin{aligned}
& N_{*} U\left(x_{0}\right) \equiv \sup _{(x, t) \in \gamma\left(x_{0}\right)}|U(x, t)|, \\
& \widetilde{N}_{*} U\left(x_{0}\right) \equiv \sup _{(x, t) \in \gamma\left(x_{0}\right)}\left(f f_{|x-y|<t}|U(y, s)|^{2} d y d s\right)^{\frac{1}{2}} .
\end{aligned}
$$

Our main result is the following:

Theorem 1.3. Suppose that $L$ is an operator of the type described above, with

$$
\left\|A-A_{0}\right\|_{\infty} \leq \epsilon
$$

for some real, symmetric, $L^{\infty}$, elliptic, and $t$-independent matrix $A_{0}$. Suppose also that $L u=0$, and that $\mathbf{v} \in W_{\text {loc }}^{1,2}\left(\mathbb{R}^{n+1}, \mathbb{C}^{n+1}\right)$. If $\epsilon \leq \epsilon_{0}$, with $\epsilon_{0}$ sufficiently small, depending only on dimension and ellipticity, then we have the bilinear estimate

$$
\left|\iint_{\mathbb{R}_{+}^{n+1}} \nabla u \cdot \overline{\mathbf{v}} d x d t\right| \leq C \sup _{t>0}\|u(\cdot, t)\|_{L^{2}\left(\mathbb{R}^{n}\right)}\left(\left\|\left|t \nabla \mathbf{v}\|\mid+\| N_{*} \mathbf{v} \|_{L^{2}\left(\mathbb{R}^{n}\right)}\right),\right.\right.
$$

where $C=C(n, \lambda, \Lambda)$ and

$$
\left\||F \|| \equiv\left(\iint_{\mathbb{R}_{+}^{n+1}}|F(x, t)|^{2} t^{-1} d x d t\right)^{1 / 2} .\right.
$$

We remark that the result is new even in the case of real, symmetric coefficients. The analogous result was proved by Dahlberg for harmonic functions in Lipschitz graph domains $\left\{(x, t) \in \mathbb{R}^{n} \times(-\infty, \infty): t>\varphi(x)\right\}$, using a special change of variable found by Kenig and Stein, and independently by Maz'ya. Our theorem includes that of Dahlberg, as may be seen by pulling back under the mapping $(x, t) \rightarrow(x, \varphi(x)+t)$. Dahlberg's original method seems inapplicable to the variable coefficient case, unless the coefficients are differentiable and satisfy an appropriate sort of Carleson condition as in the work of Kenig and Pipher [13. In the present setting, in lieu of the special change of variable, we use recently obtained results of [1] concerning the boundedness and invertibility of layer potentials associated to variable coefficient $t$-independent operators. 
The paper is organized as follows. In the next section, we recall some of the aforementioned results of [1. In Section [3, we prove Theorem 1.3, and in Section 4, we discuss the analogue of another result of [6] concerning the domain of the infinitesimal generator of the Poisson semigroup for the equation $L u=0$ in $\mathbb{R}_{+}^{n+1}$.

Let us now set some notation and terminology that we shall use in the sequel. We shall employ the standard convention that the generic constant $C$ is allowed to vary from one instance to the next, and may depend upon dimension and ellipticity. The symbol $f$ denotes the mean value, i.e., $f_{E} f \equiv|E|^{-1} \int_{E} f$. We shall use the notation

$$
D_{j} \equiv \partial_{x_{j}}, \quad 1 \leq j \leq n+1,
$$

bearing in mind that $x_{n+1}=t$, and we use $e_{j}, 1 \leq j \leq n+1$, to indicate the standard unit basis vector in the $x_{j}$ direction. The symbol $\nabla$ denotes the full $(n+1)$-dimensional gradient, acting in both $x$ and $t$, and we use $\nabla_{x}$ or $\nabla_{\|}$to indicate the $n$-dimensional gradient acting only in $x$. We use adj to denote the Hermitian adjoint of an operator acting on functions defined on $\mathbb{R}^{n}$. We define the homogeneous Sobolev space $\dot{L}_{1}^{2}$ to be the completion of $C_{0}^{\infty}$ with respect to the seminorm $\|\nabla F\|_{2}$. As is well known, for $n \geq 2$, this space can be identified (modulo constants) with the space $I_{1}\left(L^{2}\right) \equiv \Delta^{-1 / 2}\left(L^{2}\right)$. We write $F \rightarrow f$ n.t. to mean that for a.e. $x \in \mathbb{R}^{n}, F(y, t) \rightarrow f(x)$, as $(y, t) \rightarrow(x, 0)$, with $(y, t) \in \gamma(x)$.

\section{RESUltS FOR VARIABLE COEFFICIENT LAYER POTENTIALS}

We now recall the definitions of the layer potentials. We first note that by (1.4), the stability result of [2], and the classical De Giorgi-Nash Theorem [7, 15], solutions of $L u=0$ are locally Hölder continuous. Let $\Gamma(x, t, y, s)$ denote the fundamental solution for $L$ (we refer the reader to 9] for the construction of, and estimates for, $\Gamma$ in the case of complex coefficients, assuming De Giorgi-Nash bounds). By $t$-independence,

$$
\Gamma(x, t, y, s) \equiv \Gamma(x, t-s, y, 0) .
$$

We define the single and double layer potentials, respectively, in the usual way:

$$
\begin{aligned}
S_{t} f(x) & \equiv \int_{\mathbb{R}^{n}} \Gamma(x, t, y, 0) f(y) d y, \quad t \in \mathbb{R}, \\
\mathcal{D}_{t} f(x) & \equiv \int_{\mathbb{R}^{n}} \overline{\partial_{\nu^{*}(y)} \Gamma^{*}(y, 0, x, t)} f(y) d y, \quad t \neq 0,
\end{aligned}
$$

where $\partial_{\nu^{*}}$ is the adjoint exterior conormal derivative; i.e., if $A^{*}$ denotes the Hermitian adjoint of $A$, then

$$
\begin{aligned}
\partial_{\nu^{*}(y)} \Gamma^{*}(y, 0, x, t) & =-\sum_{j=1}^{n+1} A_{n+1, j}^{*}(y) \frac{\partial \Gamma^{*}}{\partial y_{j}}(y, 0, x, t) \\
& =-\left.e_{n+1} \cdot A^{*}(y) \nabla_{y, s} \Gamma^{*}(y, s, x, t)\right|_{s=0}
\end{aligned}
$$

(recall that $\left.y_{n+1}=s\right)$, where $e_{n+1} \equiv(0, \ldots, 0,1)$ is the unit basis vector in the $t$ direction. Here, $\Gamma^{*}$ is the fundamental solution for $L^{*}$, the Hermitian adjoint of $L$. Thus, $\Gamma^{*}$ is the conjugate transpose of $\Gamma$; i.e.,

$$
\overline{\Gamma^{*}(y, s, x, t)}=\Gamma(x, t, y, s) \text {. }
$$


We also define (formally) the boundary singular integral

$$
K f(x) \equiv p \cdot v \cdot \int_{\mathbb{R}^{n}} \overline{\partial_{\nu^{*}} \Gamma^{*}(y, 0, x, 0)} f(y) d y .
$$

(For the precise definiton of the latter operator in the case of non-smooth coefficients, see [1, Section 4.) In a departure from tradition impelled by the context of complex coefficients, $K^{*}, S^{*}$ and $\mathcal{D}^{*}$ will denote the analogues of $K, S$ and $\mathcal{D}$ corresponding to $L^{*}$.

In order to prove our theorem, we shall require some of the main results of [1], which we summarize as follows:

Theorem 2.5 (1]). Suppose that L satisfies the hypotheses of Theorem 1.3. There exists a small constant $\epsilon_{0}=\epsilon_{0}(n, \lambda, \Lambda)$ such that if $\epsilon$ in (1.4) satisfies $\epsilon \leq \epsilon_{0}$, then the layer potential operators $\pm \frac{1}{2} I+K, \pm \frac{1}{2} I+K^{*}$ are isomorphisms on $L^{2}\left(\mathbb{R}^{n}\right)$, with the implicit constants depending only upon dimension and ellipticity. In addition,

$$
\sup _{t>0}\left\|\mathcal{D}_{t} f\right\|_{2}+\left\|t \nabla \partial_{t} S_{ \pm t} f\right\|\left|+\sup _{1 \leq j \leq n}\left\|\mid t \partial_{t} S_{ \pm t} D_{j} f\right\| \leq C\|f\|_{2},\right.
$$

and $\mathcal{D}_{ \pm t} f \rightarrow\left(\mp \frac{1}{2} I+K\right) f$ n.t. and in $L^{2}$, for $f \in L^{2}$. Moreover, the corresponding statements hold also for $\mathcal{D}_{t}^{*}, K^{*}$ and $S_{t}^{*}$. Finally, the solution to the Dirichlet problem

$$
\left\{\begin{array}{l}
L u=0 \text { in } \mathbb{R}_{+}^{n+1}=\left\{(x, t) \in \mathbb{R}^{n} \times(0, \infty)\right\} \\
\lim _{t \rightarrow 0} u(\cdot, t)=f \text { in } L^{2}\left(\mathbb{R}^{n}\right) \text { and n.t. } \\
\sup _{t>0}\|u(\cdot, t)\|_{L^{2}\left(\mathbb{R}^{n}\right)}<\infty,
\end{array}\right.
$$

which exists by virtue of the aforementioned facts about layer potentials, is unique.

We shall also require the following technical facts.

Lemma 2.7 ([1, Lemma 2.2]). Suppose that L satisfies the hypotheses of Theorem 1.3 , with $\epsilon \leq \epsilon_{0}$. Set $K_{t}(x, y)=t \partial_{t}^{2} \Gamma(x, t, y, 0)$. Then

$$
\left|K_{t}(x, y)\right| \leq C \frac{t}{(t+|x-y|)^{n+1}} .
$$

Lemma 2.9 ([1, Lemma 2.8]). Suppose that L satisfies the hypotheses of Theorem 1.3 , with $\epsilon \leq \epsilon_{0}$. Then

$$
\left\|t^{2} \nabla \partial_{t}^{2} S_{ \pm t}^{*} f\right\|_{L^{2}\left(\mathbb{R}^{n}\right)} \leq C\|f\|_{2} .
$$

Lemma 2.10. Suppose that $\left\{R_{t}\right\}_{t>0}$ is a family of operators defined by

$$
R_{t} f(x) \equiv \int_{\mathbb{R}^{n}} K_{t}(x, y) f(y) d y,
$$

where the kernel $K_{t}$ satisfies (2.8). Suppose also that $R_{t} 1=0$ for all $t \in \mathbb{R}$. Then for $h \in \dot{L}_{1}^{2}\left(\mathbb{R}^{n}\right)$,

$$
\int_{\mathbb{R}^{n}}\left|R_{t} h\right|^{2} \leq C t^{2} \int_{\mathbb{R}^{n}}\left|\nabla_{x} h\right|^{2} .
$$

The proof of the last lemma is a standard exercise in the use of Poincaré's inequality. We omit the details, but see, e.g. 1] (Lemma 3.5), for a more general result.

Finally, we shall use the following special case of the "Fatou Theorem" of [1]. 
Lemma 2.12 ([1, Corollary 4.41]). Suppose that L satisfies the hypotheses of Theorem [1.3, with $\epsilon$ sufficiently small, and that $L u=0$ in $\mathbb{R}^{n+1}$. Suppose also that

$$
\sup _{t>0}\|u(\cdot, t)\|_{2}<\infty \text {. }
$$

Then $u(\cdot, t)$ converges n.t. and in $L^{2}$ as $t \rightarrow 0$.

\section{Proof of Theorem 1.3}

The proof of the theorem will use the following:

Lemma 3.1. Suppose that L satisfies the hypotheses of Theorem [1.3, with $\epsilon$ sufficiently small. Suppose also that $L u=0$ in $\mathbb{R}^{n+1}$, with $\sup _{t>0}\|u(\cdot, t)\|_{2}<\infty$. Then

$$
\left\|\left|t \nabla u\left\|\mid \leq C \sup _{t>0}\right\| u(\cdot, t) \|_{2} .\right.\right.
$$

Proof. It is enough to show that for each fixed $\eta \in\left(0,10^{-10}\right)$,

$$
f_{\eta}^{2 \eta} \int_{\delta}^{1 / \delta} \int_{\mathbb{R}^{n}}|\nabla u|^{2} t d x d t d \delta \leq C \sup _{t>0}\|u(\cdot, t)\|_{2} .
$$

Integrating by parts in $t$ on the left side of the last inequality, we obtain

$$
-\Re e f_{\eta}^{2 \eta} \int_{\delta}^{1 / \delta}\left\langle\partial_{t} \nabla u, \nabla u\right\rangle t^{2} d t d \delta+\text { boundary, }
$$

where the boundary terms are dominated by

$$
\sup _{r>0} f_{r}^{2 r} \int_{\mathbb{R}^{n}} r^{2}|\nabla u(x, t)|^{2} d x d t \leq C \sup _{t>0}\|u(\cdot, t)\|_{2}^{2},
$$

as desired, and in the last step we have split $\mathbb{R}^{n}$ into cubes of side length $\approx r$ and used Caccioppoli's inequality. By Cauchy's inequality, the main term in (3.2) is no larger than

$$
\frac{\varepsilon}{2} f_{\eta}^{2 \eta} \int_{\delta}^{1 / \delta} \int_{\mathbb{R}^{n}}|\nabla u|^{2} t d x d t d \delta+\frac{1}{2 \varepsilon} f_{\eta}^{2 \eta} \int_{\delta}^{1 / \delta} \int_{\mathbb{R}^{n}}\left|\nabla \partial_{t} u\right|^{2} t^{3} d x d t d \delta \equiv I+I I,
$$

where $\varepsilon>0$ is at our disposal. Choosing $\varepsilon$ small, we may hide term $I$. Having fixed $\varepsilon$, and applying Caccioppoli's inequality in Whitney boxes, we obtain that

$$
I I \leq C \iint_{\mathbb{R}_{+}^{n+1}}\left|\partial_{t} u\right|^{2} d x t d t .
$$

By the Fatou Theorem of [1], Section $4, u$ converges in $L^{2}\left(\mathbb{R}^{n}\right)$ to some $f$, with

$$
\|f\|_{2} \leq \sup _{t>0}\|u(\cdot, t)\|_{2} .
$$

Thus, by Theorem [2.5 $u(\cdot, t)=\mathcal{D}_{t}\left(-\frac{1}{2} I+K\right)^{-1} f$. By (2.6), the bijectivity of $\left(-\frac{1}{2} I+K\right)$ and the definition of $\mathcal{D}_{t}$, we obtain that

$$
\iint_{\mathbb{R}_{+}^{n+1}}\left|\partial_{t} u\right|^{2} d x t d t \leq C\|f\|_{2} \leq C \sup _{t>0}\|u(\cdot, t)\|_{2} .
$$


Proof of Theorem 1.3, By the previous lemma, it is enough to establish the bound

$$
\begin{aligned}
& \sup _{0<\eta<10^{-10}} f_{\eta}^{2 \eta}\left|\int_{\delta}^{1 / \delta} \int_{\mathbb{R}^{n}} \nabla u \cdot \overline{\mathbf{v}} d x d t\right| d \delta \\
& \quad \leq C\left(\left\|\left|t \nabla u\left\|\mid+\sup _{t>0}\right\| u(\cdot, t) \|_{L^{2}\left(\mathbb{R}^{n}\right)}\right)\left(\|t \nabla \mathbf{v}\| \mid+\sup _{t>0}\|\mathbf{v}(\cdot, t)\|_{L^{2}\left(\mathbb{R}^{n}\right)}\right) .\right.\right.
\end{aligned}
$$

We may suppose that the right-hand side of (3.4) is finite; otherwise there is nothing to prove. On the left-hand side of (3.4), for each fixed $\eta$, we integrate by parts in $t$ to obtain the bound

$$
f_{\eta}^{2 \eta}\left|\int_{\delta}^{1 / \delta} \int_{\mathbb{R}^{n}} \nabla u \cdot \overline{\partial_{t} \mathbf{v}} d x t d t\right| d \delta+f_{\eta}^{2 \eta}\left|\int_{\delta}^{1 / \delta} \int_{\mathbb{R}^{n}} \nabla \partial_{t} u \cdot \overline{\mathbf{v}} d x t d t\right| d \delta+\text { boundary, }
$$

where the boundary terms are dominated by

$$
\begin{aligned}
C\left(\sup _{r>0} f_{r}^{2 r} \int_{\mathbb{R}^{n}} r^{2}|\nabla u(x, t)|^{2} d x d t\right)^{1 / 2} & \left(\sup _{t>0}\|\mathbf{v}(\cdot, t)\|_{2}\right) \\
& \leq C\left(\sup _{t>0}\|u(\cdot, t)\|_{2}\right)\left(\sup _{t>0}\|\mathbf{v}(\cdot, t)\|_{2}\right),
\end{aligned}
$$

and we have used (3.3) in the last step. Moreover, by Cauchy-Schwarz, the first term in (3.5) is no larger than $\|t \nabla u\|\|\||t \nabla \mathbf{v} \||$.

It therefore remains to treat the middle term in (3.5). To this end, we write $\nabla=\nabla_{x}+\partial_{t} e_{n+1}$, and $\mathbf{v}=\mathrm{v}_{\|}+\mathrm{v}_{n+1} e_{n+1}$, where $v_{n+1} \equiv \mathbf{v} \cdot e_{n+1}$. Now,

$$
\begin{aligned}
& f_{\eta}^{2 \eta}\left|\int_{\delta}^{1 / \delta} \int_{\mathbb{R}^{n}} \nabla_{x} \partial_{t} u \cdot \overline{\mathrm{v}_{\|}} d x t d t\right| d \delta \\
&=f_{\eta}^{2 \eta}\left|\int_{\delta}^{1 / \delta} \int_{\mathbb{R}^{n}} \partial_{t} u \operatorname{div}_{x} \overline{\mathrm{v}_{\|}} d x t d t\right| d \delta \leq C\||t \nabla u\|\mid\| t \nabla \mathbf{v}\|\|,
\end{aligned}
$$

as desired. Thus, it is enough to consider

$$
\begin{array}{r}
f_{\eta}^{2 \eta}\left|\int_{\delta}^{1 / \delta} \int_{\mathbb{R}^{n}} \partial_{t}^{2} u \overline{\mathrm{v}_{n+1}} d x t d t\right| d \delta \leq \frac{1}{2} f_{\eta}^{2 \eta}\left|\int_{\delta}^{1 / \delta} \int_{\mathbb{R}^{n}} \partial_{t}^{3} u \overline{\mathrm{v}_{n+1}} d x t^{2} d t\right| d \delta \\
+\frac{1}{2} f_{\eta}^{2 \eta}\left|\int_{\delta}^{1 / \delta} \int_{\mathbb{R}^{n}} \partial_{t}^{2} u \overline{\partial_{t} \mathrm{v}_{n+1}} d x t^{2} d t\right| d \delta+\mathcal{B} \equiv I+I I+\mathcal{B},
\end{array}
$$

where we have again integrated by parts in $t$ and $\mathcal{B}$ denotes boundary terms which satisfy

$$
\begin{aligned}
\mathcal{B} \leq C\left(\sup _{r>0} f_{r}^{2 r} \int_{\mathbb{R}^{n}} r^{4}\left|\partial_{t}^{2} u(x, t)\right|^{2} d x d t\right)^{1 / 2} & \left(\sup _{t>0}\|\mathbf{v}(\cdot, t)\|_{2}\right) \\
\leq & C\left(\sup _{t>0}\|u(\cdot, t)\|_{2}\right)\left(\sup _{t>0}\|\mathbf{v}(\cdot, t)\|_{2}\right)
\end{aligned}
$$

by a double application of Caccioppoli's inequality. Moreover,

$$
I I \leq C\left\|\left|t ^ { 2 } \partial _ { t } ^ { 2 } u \left\|\left|\left\|\left|t \partial _ { t } \mathbf { v } \left\|\left|\leq C\left\|\left|t \partial _ { t } u \left\|\left|\left\|\mid t \partial_{t} \mathbf{v}\right\|,\right.\right.\right.\right.\right.\right.\right.\right.\right.\right.\right.\right.
$$


where we have used Caccioppoli in Whitney boxes to bound the first factor. Turning to the main term, we have that

$$
I=C f_{\eta}^{2 \eta}\left|\int_{\delta / 2}^{1 /(2 \delta)} \int_{\mathbb{R}^{n}} \partial_{t}^{3} u(x, 2 t) \overline{\mathrm{v}_{n+1}(x, 2 t)} d x t^{2} d t\right| d \delta,
$$

where we have made the change of variable $t \rightarrow 2 t$. For $t$ momentarily fixed, set

$$
g_{t}(x) \equiv \partial_{t} u(x, t) .
$$

By Theorem 2.5] (i.e., the result of [1]), we have that

$$
\tilde{u}_{t}(\cdot, s) \equiv \mathcal{D}_{s}\left(-\frac{1}{2} I+K\right)^{-1} g_{t}
$$

is the unique solution of (D2) with data $g_{t}$. Hence, by $t$-independence,

$$
\tilde{u}_{t}(\cdot, s)=\partial_{t} u(\cdot, t+s) .
$$

Setting $s=t$, we therefore obtain that

$$
\left(D_{n+1}^{3} u\right)(\cdot, 2 t)=\left(\partial_{t}^{2} \mathcal{D}_{t}\right)\left(-\frac{1}{2} I+K\right)^{-1} g_{t} .
$$

We observe that by (2.1), (2.2) and (2.3),

$$
\operatorname{adj}\left(\partial_{t}^{2} \mathcal{D}_{t}\right)=\partial_{\nu^{*}} \partial_{t}^{2} S_{-t}^{*} .
$$

Consequently, (3.7) becomes

$$
I=C f_{\eta}^{2 \eta}\left|\int_{\delta / 2}^{1 /(2 \delta)} \int_{\mathbb{R}^{n}}\left(-\frac{1}{2} I+K\right)^{-1} \partial_{t} u(\cdot, t) \overline{\partial_{\nu^{*}} D_{n+1}^{2} S_{-t}^{*} \mathrm{v}_{n+1}(\cdot, 2 t)} d x t^{2} d t\right| d \delta,
$$

so by Cauchy-Schwarz and Theorem 2.5, it suffices to prove

$$
\left\|\left|t^{2} \nabla D_{n+1}^{2} S_{-t}^{*} \mathbf{v}(\cdot, 2 t) \|\right| \leq C\left(\left\|\left|t \nabla \mathbf{v}\|\mid+\| N_{*} \mathbf{v} \|_{2}\right) .\right.\right.\right.
$$

The left-hand side of (3.8) equals

$$
\begin{aligned}
& \left(\sum_{k=-\infty}^{\infty} \int_{2^{k}}^{2^{k+1}} \int_{\mathbb{R}^{n}}\left|t^{2} \nabla D_{n+1}^{2} S_{-t}^{*} \mathbf{v}(\cdot, 2 t)\right|^{2} \frac{d x d t}{t}\right)^{1 / 2} \\
& \leq\left(\sum_{k=-\infty}^{\infty} \int_{2^{k}}^{2^{k+1}} \int_{\mathbb{R}^{n}}\left|t^{2} \nabla D_{n+1}^{2} S_{-t}^{*}\left(\mathbf{v}(\cdot, 2 t)-\mathbf{v}\left(\cdot, 2 t_{k}\right)\right)\right|^{2} \frac{d x d t}{t}\right)^{1 / 2} \\
& \quad+\left(\sum_{k=-\infty}^{\infty} \int_{2^{k}}^{2^{k+1}} \int_{\mathbb{R}^{n}}\left|t^{2} \nabla D_{n+1}^{2} S_{-t}^{*} \mathbf{v}\left(\cdot, 2 t_{k}\right)\right|^{2} \frac{d x d t}{t}\right)^{1 / 2} \equiv I I I+I V,
\end{aligned}
$$


where $t_{k}=2^{k-1}$. We consider term $I V$ first. Dividing $\mathbb{R}^{n}$ into cubes of side length $2^{k}$ and using Caccioppoli's inequality, we deduce that

$$
\begin{aligned}
I V \leq & C\left(\sum_{k=-\infty}^{\infty} \int_{2^{k-1}}^{2^{k+2}} \int_{\mathbb{R}^{n}}\left|t D_{n+1}^{2} S_{-t}^{*} \mathbf{v}\left(\cdot, 2 t_{k}\right)\right|^{2} \frac{d x d t}{t}\right)^{1 / 2} \\
\leq & C\left(\sum_{k=-\infty}^{\infty} \int_{2^{k-1}}^{2^{k+2}} \int_{\mathbb{R}^{n}}\left|t D_{n+1}^{2} S_{-t}^{*}\left(\mathbf{v}\left(\cdot, 2 t_{k}\right)-\mathbf{v}(\cdot, 2 t)\right)\right|^{2} \frac{d x d t}{t}\right)^{1 / 2} \\
& +\left\|\left|( t D _ { n + 1 } ^ { 2 } S _ { - t } ^ { * } 1 ) ( P _ { t } \mathbf { v } ( \cdot , 2 t ) ) \left\|\left|+\left\|\left|R_{t} \mathbf{v}(\cdot, 2 t) \|\right| \equiv I V_{1}+I V_{2}+I V_{3},\right.\right.\right.\right.\right.
\end{aligned}
$$

where

$$
R_{t} \equiv t D_{n+1}^{2} S_{-t}^{*}-\left(t D_{n+1}^{2} S_{-t}^{*} 1\right) P_{t}
$$

and $P_{t}$ is a nice approximate identity with a smooth, compactly supported kernel. By Lemma 2.10,

$$
I V_{3} \leq\||t \nabla \mathbf{v} \|| .
$$

By (2.6), Lemma 2.7, and a well-known argument of Fefferman and Stein [8], we have that $\left|t \partial_{t}^{2} S_{-t}^{*} 1\right|^{2} \frac{d x d t}{t}$ is a Carleson measure, whence

$$
I V_{2} \leq C\left\|N_{*} \mathbf{v}\right\|_{2} \text {. }
$$

By Lemma 2.7, the operator $f \rightarrow t D_{n+1}^{2} S_{-t}^{*} f$ is bounded on $L^{2}\left(\mathbb{R}^{n}\right)$, so that

$$
\begin{aligned}
& I V_{1} \leq C\left(\sum_{k=-\infty}^{\infty} \int_{2^{k-1}}^{2^{k+2}} \int_{\mathbb{R}^{n}}\left|\frac{1}{\sqrt{t}} \int_{2 t_{k}}^{2 t} \partial_{s} \mathbf{v}(x, s) d s\right|^{2} d x d t\right)^{1 / 2} \\
& \leq C\left(\sum_{k=-\infty}^{\infty} \iint_{\mathbb{R}^{n+1}}\left|f_{2 t_{k}}^{2 t} 1_{\left\{2^{k} \leq s \leq 2^{k+3}\right\}} \sqrt{s} \partial_{s} \mathbf{v}(x, s) d s\right|^{2} d x d t\right)^{1 / 2} \leq C\left\|\mid t \partial_{t} \mathbf{v}\right\| \|,
\end{aligned}
$$

where in the last step we have used the boundedness of the Hardy-Littlewood maximal function.

Finally, we consider term III in (3.9). By Lemma 2.9. III may be handled like $I V_{1}$ above. We omit the details.

\section{The domain of the generator of the Poisson semigroup}

In this section we generalize to our setting a result of [6] concerning the domain of the generator of the Poisson semigroup. We continue to suppose that the hypotheses of Theorem 1.3 hold. By Theorem 2.5, if $\epsilon \leq \epsilon_{0}$ is sufficiently small, then the Dirichlet problem (D2) has a unique solution. Consequently, the solution operator $f \rightarrow \mathcal{P}(t) f \equiv u(\cdot, t)$, where $u$ solves (D2) with data $f$, satisfies

$$
\sup _{t>0}\|\mathcal{P}(t)\|_{2 \rightarrow 2} \leq C, \quad \lim _{t \rightarrow 0}\|\mathcal{P}(t) f-f\|_{2}=0
$$

and

$$
\mathcal{P}(t+s)=\mathcal{P}(t) \mathcal{P}(s),
$$

where the last identity also uses $t$-independence of the coefficients. Standard semigroup theory therefore implies that the semigroup $\{\mathcal{P}(t)\}$ has a densely defined infinitesimal generator on $L^{2}\left(\mathbb{R}^{n}\right)$, which we denote by $\mathcal{A}$. We will show that the domain $D(\mathcal{A})$ of this generator is the Sobolev space $L_{1}^{2} \equiv L^{2} \cap \dot{L}_{1}^{2}$. More precisely, we have the following: 
Theorem 4.3. Suppose that $L$ is as above. Then $D(\mathcal{A})=L_{1}^{2}$, and

$$
\|\mathcal{A} f\|_{2} \approx\left\|\nabla_{x} f\right\|_{2} \text {. }
$$

We remark that this last theorem can be viewed as an extension of the Kato square root problem $([\mathbf{5},[11,[4,[10$ and $[3])$ to the case that the coefficient matrix $A$ is a full $(n+1) \times(n+1)$ matrix. Indeed, the Kato problem corresponds to the case that the coefficient matrix has the special "block" structure

$$
\left[\begin{array}{c|c} 
& 0 \\
B & \vdots \\
& 0 \\
\hline 0 \cdots 0 & 1
\end{array}\right]
$$

where $B=B(x)$ is an $n \times n$ matrix. In the latter case the generator of the Poisson semigroup is

$$
-\sqrt{-\operatorname{div}_{x} B \nabla_{x}}
$$

and the conclusion of Theorem 4.3 is the (now established) Kato conjecture.

We also note that by Theorem 2.5. we have the representation

$$
\mathcal{P}(t)=\mathcal{D}_{t}\left(-\frac{1}{2} I+K\right)^{-1} .
$$

In order to prove the theorem we shall require the following result from [1.

Theorem 4.6 (11). Suppose that $L$ satisfies the hypotheses of Theorem 1.3 . There exists a small constant $\epsilon_{0}=\epsilon_{0}(n, \lambda, \Lambda)$ such that if $\epsilon$ in (1.4) satisfies $\epsilon \leq \epsilon_{0}$, then the single layer potential satisfies

$$
\sup _{t \in \mathbb{R}}\left\|\nabla S_{t}\right\|_{2 \rightarrow 2} \leq C,
$$

and $\left.S_{0} \equiv S_{t}\right|_{t=0}: L^{2}\left(\mathbb{R}^{n}\right) \rightarrow \dot{L}_{1}^{2}\left(\mathbb{R}^{n}\right)$ is a bijection. Moreover, there is a unique solution to the regularity problem

$$
\left\{\begin{array}{l}
L u=0 \text { in } \mathbb{R}_{+}^{n+1} \\
u(\cdot, t) \rightarrow f \in \dot{L}_{1}^{2}\left(\mathbb{R}^{n}\right) \text { n.t. } \\
\widetilde{N}_{*}(\nabla u) \in L^{2}\left(\mathbb{R}^{n}\right)
\end{array}\right.
$$

which has the representation

$$
u(\cdot, t) \equiv S_{t}\left(S_{0}^{-1} f\right),
$$

and $\partial_{t} u(\cdot, t)$ converges n.t. and in $L^{2}\left(\mathbb{R}^{n}\right)$ as $t \rightarrow 0$. Finally,

$$
\left.\left(\nabla S_{t}\right)\right|_{t= \pm s} f \rightarrow \mp \frac{1}{2} \cdot \frac{f(x)}{A_{n+1, n+1}(x)} e_{n+1}+\mathcal{T} f
$$

weakly in $L^{2}\left(\mathbb{R}^{n}\right)$, where $\mathcal{T}: L^{2}\left(\mathbb{R}^{n}\right) \rightarrow L^{2}\left(\mathbb{R}^{n}, \mathbb{C}^{n+1}\right.$ ) (see 1 , Lemma 4.18 for a precise definition of $\mathcal{T}$ ).

Proof of Theorem 4.3. The deep results underlying Theorem 4.3 are Theorems 2.5 and 4.6, and we shall deduce the first as a straightforward corollary of the latter two. We observe that if $u$ solves (R2) with data $f \in L_{1}^{2}$, then

$$
\lim _{t \rightarrow 0} \partial_{t} u(\cdot, t)=\lim _{t \rightarrow 0} \partial_{t} \mathcal{P}(t) f \equiv \mathcal{A} f .
$$


Thus, by (4.7), (4.8) and the bijectivity of $S_{0}$,

$$
\|\mathcal{A} f\|_{2} \leq C\left\|\nabla_{x} f\right\|_{2} .
$$

The proof of the opposite inequality is only a bit harder, and we sketch the details briefly here. We modify slightly the strategy of Verchota [16. By the well-known Rellich identity (see, e.g., [12]) and the case $\epsilon=0$ of Theorem 4.6, for $A_{0}$ real and symmetric we have that

$$
\left\|\nabla_{x} u_{0}(\cdot, t)\right\|_{2} \approx\left\|\partial_{t} u_{0}(\cdot, t)\right\|_{2},
$$

uniformly in $t \geq 0$, when $u_{0}(\cdot, t) \equiv S_{t}^{0} f$, and $S_{t}^{0}$ is the single layer potential associated to $L_{0} \equiv-\operatorname{div} A_{0} \nabla$. By Theorem 4.6 and analytic perturbation theory,

$$
\left\|\left(\nabla S_{t}^{0}-\nabla S_{t}\right) f\right\| \leq C\left\|A_{0}-A\right\|_{\infty}\|f\|_{2} .
$$

The latter estimate, combined with (4.10), yields, uniformly in $t \geq 0$,

$$
C^{-1}\left\|\partial_{t} S_{t} f\right\|_{2}-C \epsilon_{0}\|f\|_{2} \leq\left\|\nabla_{x} S_{t} f\right\|_{2} \leq C\left\|\partial_{t} S_{t} f\right\|_{2}+C \epsilon_{0}\|f\|_{2} .
$$

Since the tangential derivatives $\nabla_{x} S_{t} f$ do not jump across the boundary, the latter bound, plus its analogue for the lower half-space, and (4.9) imply

$$
\left\|\frac{1}{2}\left(A_{n+1, n+1}\right)^{-1} f+\mathcal{T}_{n+1} f\right\|_{2} \leq\left\|\frac{1}{2}\left(A_{n+1, n+1}\right)^{-1} f-\mathcal{T}_{n+1} f\right\|_{2}+C \epsilon_{0}\|f\|_{2},
$$

where $\mathcal{T}_{n+1} \equiv \mathcal{T} \cdot e_{n+1}$. Thus, by the accretivity of $A_{n+1, n+1}$ we have

$$
\begin{aligned}
\|f\|_{2} \leq\left\|\frac{1}{2}\left(A_{n+1, n+1}\right)^{-1} f+\mathcal{T}_{n+1} f\right\|_{2} & +\left\|\frac{1}{2}\left(A_{n+1, n+1}\right)^{-1} f-\mathcal{T}_{n+1} f\right\|_{2} \\
\leq C & \leq \frac{1}{2}\left(A_{n+1, n+1}\right)^{-1} f-\mathcal{T}_{n+1} f\left\|_{2}+C \epsilon_{0}\right\| f \|_{2} .
\end{aligned}
$$

For $\epsilon_{0}$ small enough, we may first hide the small term and then obtain invertibility on $L^{2}$ of $-\frac{1}{2}\left(A_{n+1, n+1}\right)^{-1} I+\mathcal{T}_{n+1}$ using (4.11) and the method of continuity as in [1. Now, given $f \in D(\mathcal{A})$, we set

$$
\tilde{u}(\cdot, t) \equiv \mathcal{P}(t) f, \quad u(\cdot, t) \equiv S_{t}\left(-\frac{1}{2}\left(A_{n+1, n+1}\right)^{-1} I+\mathcal{T}\right)^{-1} \mathcal{A} f
$$

so that $\partial_{t} \tilde{u}(\cdot, t), \partial_{t} u(\cdot, t) \rightarrow \mathcal{A}$ f n.t. and in $L^{2}$ as $t \rightarrow 0$. By uniqueness in (D2), $\partial_{t} \tilde{u}=\partial_{t} u$; hence $\tilde{u}-u$ depends only on $x$. Therefore, since $L(\tilde{u}-u)=0$ and $\tilde{u}(\cdot, t)-u(\cdot, t) \in \dot{L}_{1}^{2}$, for each fixed $t>0$ (for $\tilde{u}$, this is a consequence of the representation (4.5)), we deduce that $\tilde{u}-u=$ constant. Thus, we have that

$$
\left\|\nabla_{x} f\right\|_{2} \leq \sup _{t \geq 0}\left\|\nabla_{x} \tilde{u}(\cdot, t)\right\|_{2}=\sup _{t \geq 0}\left\|\nabla_{x} u(\cdot, t)\right\|_{2} \leq C\|\mathcal{A} f\|_{2},
$$

where in the last step we have used (4.7) and the bijectivity of $-\frac{1}{2}\left(A_{n+1, n+1}\right)^{-1} I+$ $\mathcal{T}_{n+1}$.

\section{ACKNOWLEDGEMENT}

The author thanks Zhongwei Shen for posing the question. 


\section{REFERENCES}

[1] M. Alfonseca, P. Auscher, A. Axelsson, S. Hofmann and S. Kim, Analyticity of layer potentials and $L^{2}$ solvability of boundary value problems for divergence form elliptic equations with complex $L^{\infty}$ coefficients, preprint http://www.math.missouri.edu/ ^hofmann/.

[2] P. Auscher, Regularity theorems and heat kernel for elliptic operators. J. London Math. Soc. (2) 54 (1996), no. 2, 284-296. MR1405056 (97f:35034)

[3] P. Auscher, S. Hofmann, M. Lacey, A. Mc Intosh, and P. Tchamitchian, The solution of the Kato square root problem for second order elliptic operators on $\mathbb{R}^{n}$, Annals of Math. (2) 156 (2002), 633-654. MR.1933726 (2004c:47096c)

[4] P. Auscher, S. Hofmann, J.L. Lewis, P. Tchamitchian, Extrapolation of Carleson measures and the analyticity of Kato's square-root operators, Acta Math. 187 (2001), no. 2, 161-190. MR.1879847 (2004c:47096a)

[5] R. Coifman, A. M Intosh, and Y. Meyer. L'intégrale de Cauchy définit un opérateur borné sur $L^{2}$ pour les courbes lipschitziennes. Ann. Math. (2) 116 (1982), 361-387. MR672839 (84m:42027)

[6] B. Dahlberg, Poisson semigroups and singular integrals, Proc. Amer. Math. Soc. 97 (1986), 41-48. MR831384 (87g:42035)

[7] E. De Giorgi, Sulla differenziabilità e l'analiticità delle estremali degli integrali multipli regolari (Italian). Mem. Accad. Sci. Torino. Cl. Sci. Fis. Mat. Nat. (3) 3 (1957), 25-43. MR0093649 (20:172)

[8] C. Fefferman and E. M. Stein, $H^{p}$ spaces of several variables, Acta Math. 129 (1972), no. 3-4, 137-193. MR0447953 (56:6263)

[9] S. Hofmann and S. Kim, The Green function estimates for strongly elliptic systems of second order, Manuscripta Mathematica 124 (2007), 139-172. MR.2341783

[10] S. Hofmann, M. Lacey and A. M Intosh. The solution of the Kato problem for divergence form elliptic operators with Gaussian heat kernel bounds. Annals of Math. (2) 156 (2002), pp. 623-631. MR1933725 (2004c:47096b)

[11] S. Hofmann and A. M Intosh, The solution of the Kato problem in two dimensions, Proceedings of the Conference on Harmonic Analysis and PDE held in El Escorial, Spain, in July 2000, Publ. Mat. Vol. extra, 2002, pp. 143-160. MR.1964818 (2004c:47097)

[12] C. Kenig, Harmonic analysis techniques for second order elliptic boundary value problems, CBMS Regional Conference Series in Mathematics, 83. Published for the Conference Board of the Mathematical Sciences, Washington, DC, by the American Mathematical Society, Providence, RI, 1994. MR.1282720 (96a:35040)

[13] C. Kenig and J. Pipher, The Dirichlet problem for elliptic equations with drift terms, Publ. Mat. 45 (2001), no. 1, 199-217. MR.1829584(2002e:35017)

[14] D. Mitrea, M. Mitrea and M. Taylor, Layer potentials, the Hodge Laplacian, and global boundary problems in nonsmooth Riemannian manifolds. Mem. Amer. Math. Soc. 150 (2001), no. 713. MR.1809655 (2002g:58026)

[15] J. Nash, Continuity of solutions of parabolic and elliptic equations, Amer. J. Math. 80 (1958), 931-954. MR0100158 (20:6592)

[16] G. Verchota, Layer potentials and regularity for the Dirichlet problem for Laplace's equation in Lipschitz domains. J. Funct. Anal. 59 (1984), no. 3, 572-611. MR769382 (86e:35038)

Department of Mathematics, University of Missouri, Columbia, Missouri 65211

E-mail address: hofmann@math.missouri.edu 